\title{
A String Twining Robot for High Trellis Hop Production
}

\author{
Long He ${ }^{1}$, Jianfeng Zhou ${ }^{1}$, Qin Zhang ${ }^{1 *}$, and Henry J. Charvet ${ }^{2}$ \\ ${ }^{1}$ Center for Precision and Automated Agricultural Systems, Washington State University \\ ${ }^{2}$ S.S. Steiner, Inc \\ *Corresponding author: Qin Zhang, Center for Precision and Automated Agricultural Systems, \\ Washington State University, Prosser, WA 99350, USA; Phone: 509-786-9360; Email: qinzhang@wsu.edu.
}

Abstract: The hop plant is usually trained to grow on strings in commercial production. String twining is a labor intensive task in high trellis hop fields, and there is a high demand from industry to have the operation mechanized. In this study, an innovative string twining robot, comprising end-effectors for knot tying, string feeding, and trellis wire capturing was designed to perform this task autonomously. A laboratory-scale, proof of concept prototype, was fabricated to validate the performance and effectiveness of this robotic device and associated control algorithms. Functionality assessment tests verified that the string feeding end-effector could feed $6 \mathrm{~m}$ length of string with acceptable variation. The trellis wire capturing end-effector could functionally achieve the required procedure for continuous twining. The comprehensive twining test proved that the integrated twining robot took approximately $11.2 \mathrm{~s}$ to coordinate all three end-effectors to complete one string twining cycle with a moving forward speed of the mobile platform at $0.19 \mathrm{~m} \cdot \mathrm{s}^{-1}$. At this speed, the developed prototype robot achieved $97 \%$ of successful rate. The laboratory test results indicated that the developed prototype robot has the potential to be implemented for high trellis hop twining task.

Keywords: High trellis hop, hop twining, automation, robotics, mechanization 
The State of Washington is the major hop production state in the United States, with about 9,500 ha of hops planted in 2011 accounting for 79\% US production (USDA-NASS, 2011). Almost all of the hops in Washington are grown in $5.5 \mathrm{~m}$ high trellis systems (WSDLI, 2002).

27 One essential operation in high trellis hop production is string twining on trellis wires. The twining process consists of two major tasks of tying one end of a string on an overhead trellis wire, and staking the other end into the ground. In the State of Washington, twining operation begins in April and it requires tying approximately 4,500 strings per hectare (Personal communication with growers in Wash.). Conventionally, growers in the region fasten strings on trellis wires using clove hitch knots. Figure 1 shows a typical high trellis hop field after strings has been twined. Currently, string twining is performed manually by highly trained field workers. The aging and declining availability of workers creates a major challenge to hop production. Aimed to solve the aforementioned challenges, this research was to develop a robotic solution to tie clove hitch knots on trellis wires and replace the need for human workers.

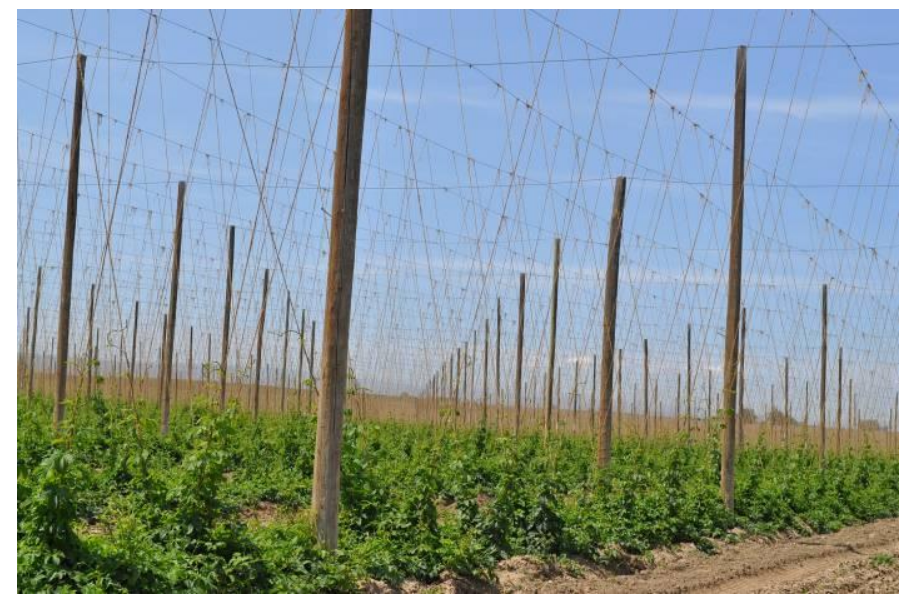

Figure 1. An example of typical high trellis hop field after being twined in the State of Washington

41 Riehm, 2003; Thomas and Jenkins, 2003; Singhatat and Corporation, 2004). The knot tying 
42 process in hop twining is quite similar to that for tying the bales. However, knots in hop twining

43 are placed on a very small size overhead wire using one string end, and the baler process using

44 two string ends putting the knots on either square or round bale for the baler (Schoonheere et al.,

45 2007). People have put efforts in the past to develop mechanical twining solutions. For example,

46 Keller Swartwood Engineering, Inc. (2003) designed and fabricated a prototype of self-propel

47 automated twining machine for attaching strings on overhead wires using hog rings. While, the

48 hog rings could not fasten the string on the trellis wires, causing the string sliding on trellis wires

49 under strong winds which commonly occur during the hop growing season. The moving of

50 strings resulted in unacceptable wearing of string worn-out and the hop vines falling down on the

51 ground. More recently, Gobor and his colleagues (Gobor and Fröhlich, 2010; Gobor et al., 2012)

52 in Germany developed a mechanical device capable of performing a fully automated twining

53 operation to fasten metal wires on trellis wires for hop production. However, Washington

54 growers prefer to use fiber string rather than the metal string. As all reported inventions could

55 not meet the need of tying clove hitch knots required by growers, researchers at Washington

56 State University (WSU) have worked closely with the hop industry to develop robotic solutions

57 for tying clove hitch knot using fiber string, and successfully invented a couple designs of knot

58 tying end-effecter capable of tying the required knots on trellis wires (He et al., 2012; He et al., 59 2013).

The work described in this paper builds upon this accomplishment towards the

61 development a robotic string twining machine. The primary goal of this study was to develop

62 complementary components to support the previous developed knot tying end-effector. To create

63 a fully automated robotic string twining machine, the components developed included a string

64 feeding end-effector and a trellis wire capturing end-effector. A series of equations were used to 
express the actions of the string feeding end-effector and the trellis wire capturing end-effector to

66 provide the baseline information for their functionality tests. Finally, a series of laboratory test

67 was conducted to evaluate the performance of the integrated robotic device, including string

68 feeding, trellis wire capturing and knot tying devices.

2 Materials and Methods

\subsection{Knot Tying Procedures}

The method of knot tying used in this study has reported in our previous study (He et al.,

72 2013). Figure 2 illustrates the step-by-step procedure of tying a clove hitch knot. In this process,

73 one end of the string is brought to the starting point 1 by a string feeding end-effector, then a

74 knot tying end-effector will grasp the string end to cross over a trellis wire twice to form two

75 loops following a pre-defined trajectory from point 1 to point 2 , then points 3 and 4 , and finally

76 pull the string end from point 4 to point 5 to complete a knot. The designated string material used

77 here was a $4 \mathrm{~mm}$ diameter plant fiber string commonly used in Washington high trellis hop

78 production.

79

80

81

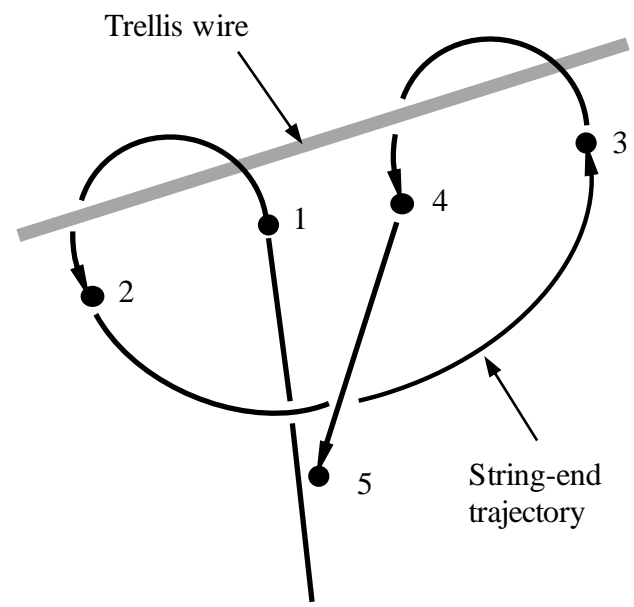

Figure 2. An illustration of the clove hitch knot tying procedure.

2.2 Design of the String Twining Robot 
83 strings are tied) and support wires (perpendicularly arranged to trellis wires to form a trellis 84 network) commonly $5.5 \mathrm{~m}$ above the ground. Generally, it requires tying four strings on one 85 section of trellis wire between two support wires to train the hop plants as illustrated in Figure 3. 86 In practice, the twining operation progresses in the direction of perpendicular to trellis wires. In 87 order to achieve the proposed goal of performing the twining operation continuously, the twining robot was designed as illustrated in Figure 4.

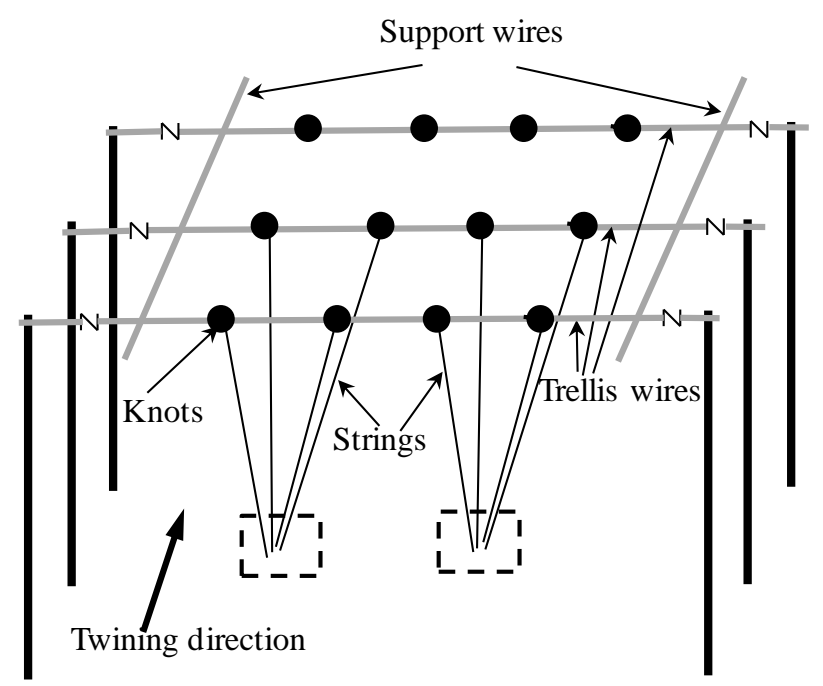

Figure 3. An illustration of trellis system and string twining positions in typical Washington high trellis hop fields. 


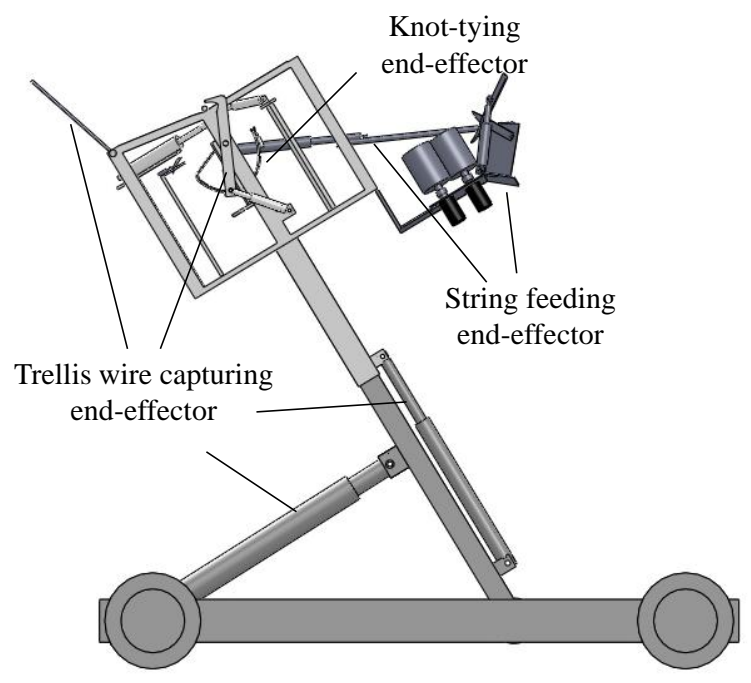

$93 \quad$ Figure 4. A structural illustration of developed string twining robot integrated by a knot tying

94 end-effector; a string feeding end-effector; and a trellis wire capturing end-effector, assembled on a mobile platform.

96

97

98

99

100

\subsubsection{Design and control of string feeding end-effector}

String with predetermined length should be placed to the designated position in a timely manner in each knot tying cycle. The detail of the string feeding end-effector is illustrated in Figure 5. Three pneumatic cylinders (elements 4, 20, and 23), and an electric motor pair (element set 21) are used in the end-effector.

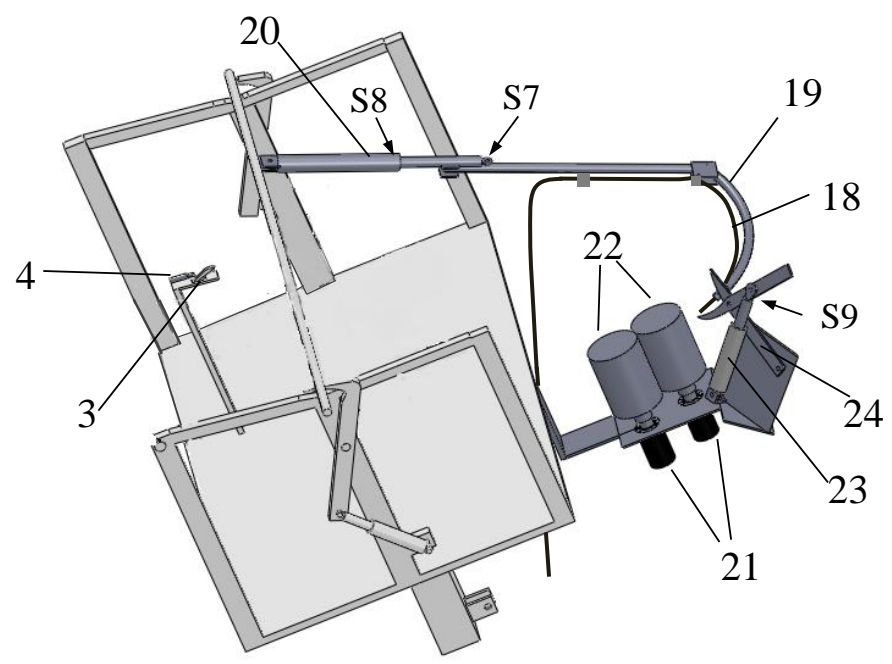


Figure 5. A structural illustration of the developed string feeding end-effector. Element 3 is a pincher; 18 represents the string to be delivered; 19 is a swing arm; 21 is an electric motor pair; 22 is a roller pair; 24 is a string cutter; and 4, 20, 23 are pneumatic cylinders.

Figure 6 shows the schematic of pneumatic actuating system of the string feeding endeffector. The actuating system used a three-way, two-position directional valves 33 (MME31NES-D024, Clippard Instruments Laboratory, Cincinnati, OH) to extend pneumatic cylinder 4 to open pincher 3 . This cylinder was retracted by an inside spring after the air pressure being released to close the pincher 3 . One four-way, three-position directional control valve (elements 32.1-32.2, MME-41NES-D024, Clippard Instruments Laboratory, Cincinnati, OH) were used to swing arm 19 forward and backward through extending or retracting cylinder 20, and another valve of the same model to drive cylinder 23 to control cutter 24 performing cutting operations. Two two-way throttle check valves 35.1 and 35.2 (JFC-4K-P08, Clippard Instruments Laboratory, Cincinnati, OH) were used to adjust the speed of cylinder 20. A pair of electric motors 21 (DED216G, DeWalt, Baltimore, MD) were used to drive a pair of rollers to feed a string with predetermined length. The string length was measured by the time of the motor pair operating in a certain rotation speed controlled using a motor controller (SyRen 10, Robotshop, Swanton, VT). In the schematic, DT9-DT13 presents the solenoids for controlling the directional valves, S7-S9 are electrical micro-switches used to provide accurate position limit for pneumatic cylinders. 
121

122 Figure 6. Schematic of the pneumatic actuating system of string feeding end-effector. DT9-DT13

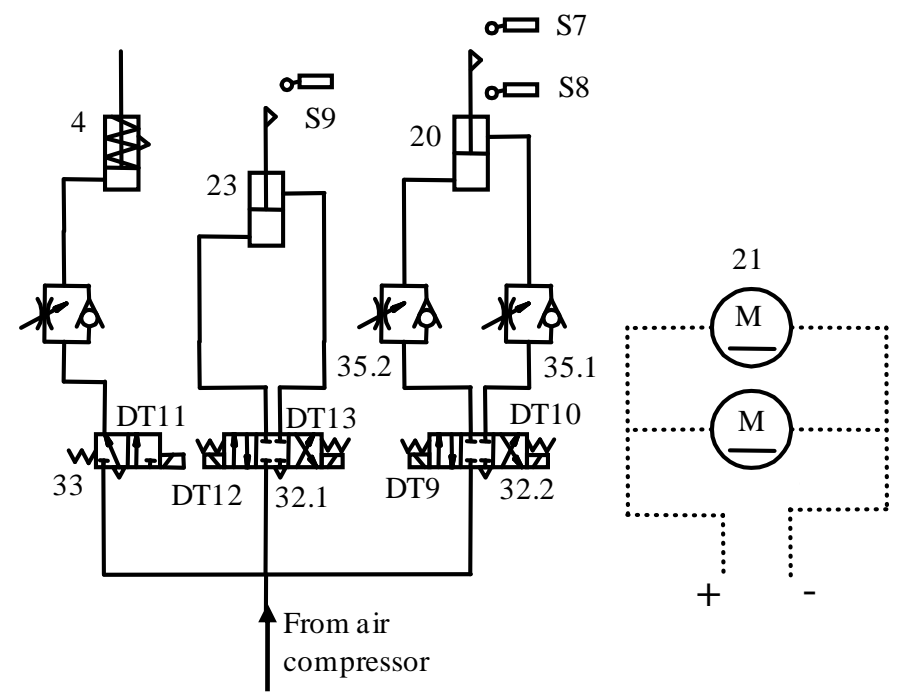
are solenoids for controlling direction control valves; S7-S9 are micro-switches to provide position limits for cylinders 20 and 23; and DC motor pair 21 is used to drive the roller pair.

A five-step procedure was designed to control this end-effector performing the string feeding operation (Figure 7). The first step was to place one end of a string (element 18 in Figure 5) on the tip of a swing arm (element 19) as the start position of string feeding. This step was automatically achieved except the manual placement for the first knotting cycle. It was followed by step two in which cylinder 20 was retracted to drive the swing arm 19 to the position of pincher 3 for delivering the string end to the pincher until switch S8 was closed (S8 “+”). At step three the system would consequently close pincher 3 to grasp the delivered string-end, then cylinder 20 pushed swing arm 19 back to its original position until it closed switch S7 (S7 “+”) after completing the delivery. In step four the electric motor pair 21 starts to run, and the string will be pushed in between the rollers 22 . A spring was used between two rollers to generate a tension, which makes the string tightly stay in between the rollers during string feeding process. The rollers 22 will stop after $5 \mathrm{~s}$ (Delay $5 \mathrm{~s}$ ) until the roller pair pulled the string to the designed 
137 length of $6 \mathrm{~m}$. Then, at step five, cylinder 23 drove the cutter 24 to cut the string, and the rollers 138 run again until the cut end of the string was fully pulled out. After completing the whole process, 139 all the components would move back to their original positions ready for the next cycle, and the 140 cut end of the string was kept on the swing tip to make it ready for the next feeding cycle.

141

142

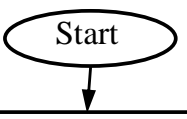

Put string end onto the swing

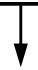

Swing rotated to pincher 3

S 8 “+”

Grasp the string end by pincher 3

and rotate back the swing

S 7 “+”

Pull string for $6 \mathrm{~m}$ by rollers

Delay $5 \mathrm{~s}$

Cut off string by cutter

S 9 “+”

End

Figure 7. Control flowchart of the string feeding end-effector. In the chart, "+” indicates closing corresponding switches.

To obtain the required string length, the rotation speed and time of the rollers needed to be determined. Figure 8 illustrates the geometric relationship between the length of fed string and the rotating speed and duration of the roller pair. In this figure, $a_{0}$ is the distance from pincher 3 to the roller; $b_{0}$ is the distance from the roller to the cutter $(\mathrm{mm}) ; \omega$ is the roller rotating speed (rpm); and $r_{0}$ is the roller radius $(\mathrm{mm})$. For this concept-proof end-effector, $a_{0}=600 \mathrm{~mm}$, $b_{0}=100 \mathrm{~mm}, \mathrm{r}_{0}=40 \mathrm{~mm}$, and $\omega=250 \mathrm{rpm}$. 


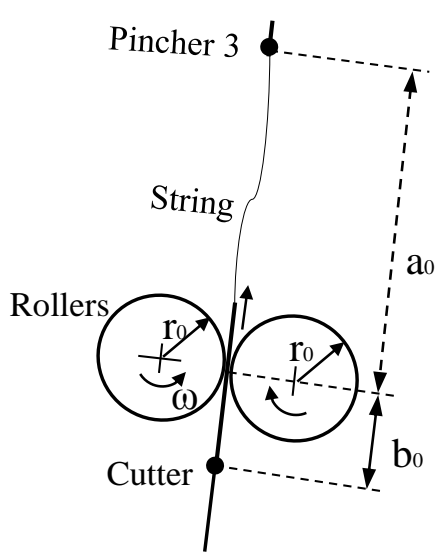

151 Figure 8. Illustration of between the length of fed string and the rotating speed of roller pair for the designed string feeding mechanism.

154 As the required length of the string is the sum of the distance between trellis wire and ground, 155 plus the length of the string for knot and sticking into the ground, the target length of the string 156 was set to $6 \mathrm{~m}$.

$$
l=a_{0}+b_{0}+2 \pi r_{0} \cdot \omega \cdot t / 60
$$

158 where, $l$ is the length of cut string segment ( $\mathrm{mm})$; and $t$ is the rolling duration (s).

159 2.2.2 Design and control of trellis wire capturing end-effector

160 The trellis wire capturing end-effector illustrated in Figure 9, is designed to keep the 161 tying end-effector in a relatively static position during the entire knot tying process. This end162 effector comprises a trellis wire detecting bar 25, two trellis wire graspers 16 and 17 controlled 163 by cylinders 14 and 15, and two position-adjusting cylinders 26 and 27, all installed on a mobile 164 platform 28 (cylinder 15 and grasper 17 are not visible in this illustration as they are placed 165 symmetrically to cylinder 14 and grasper 16 on the opposite side of the device). The trellis wire 166 detecting bar 25 was made of a rotatable metal arm which was long enough to touch trellis wires 167 as the mobile platform travelling perpendicularly to the wires. 


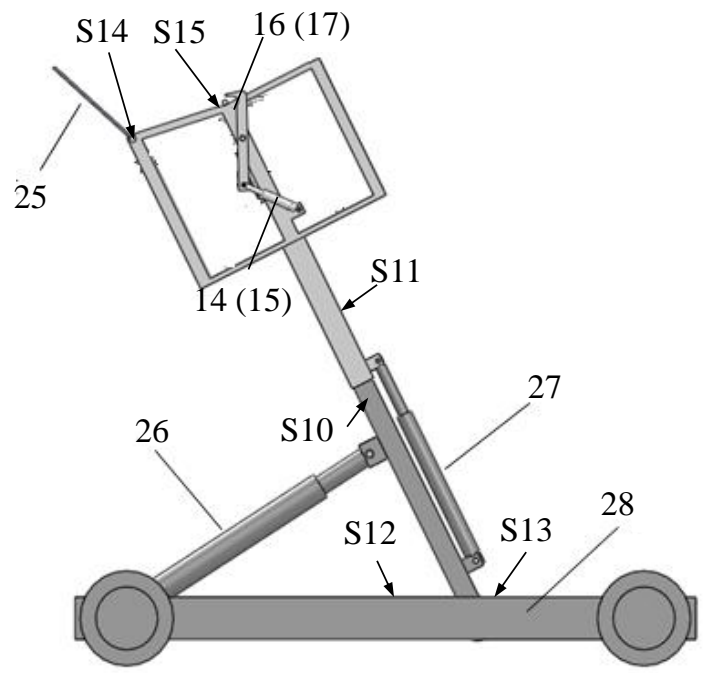

Figure 9. A structural illustration of trellis wire capturing end-effector. The end-effector comprises a metal trellis wire detecting bar 25, two trellis graspers 16 and 17; four cylinders 14, 15, 26 and 27 on a mobile platform 28. S12 to S15 are position-limit switches.

Figure 10 shows the schematic of pneumatic actuating system for this trellis wire capturing end-effector. The pneumatic system used a four-way, three-position directional control valves 32.3 (MME-41NES-D024, Clippard Instruments Laboratory, Cincinnati, OH) to control cylinders 14 and 15 for grasping or releasing trellis wire. Two more the same directional control valves were used to control cylinder 26 moving the end-effector forward and backward (valve 36.1) or control cylinder 27 lifting it up and down (valve 36.2). Four two-way throttle check valves (JFC-4K-P08, Clippard Instruments Laboratory, Cincinnati, OH) were used to control the extending and retracting speed of those cylinders. DT14-DT19 were six electric solenoids of three directional control valves (components 32.3, 36.1 and 36.2). This system used four additional limit switches (S10 to S13) to control the extending and retracting positions of Cylinders 26 and 27. 


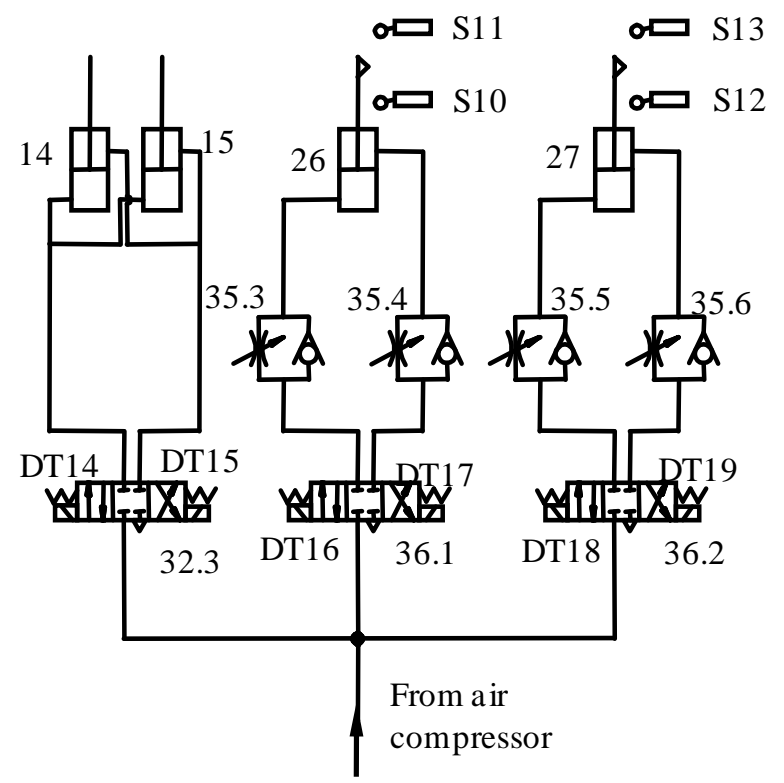

184 Figure 10. Schematic of trellis capturing end-effector pneumatic actuating system. In this 185 schematic, solenoids DT14-DT19 were used to control the directional valves; and limit switches 186 S10-S13 were used to limit the strokes of pneumatic cylinders. In this trellis wire capturing process, the wire capturing end-effector trajectory relative to 188 the mobile platform 28 was designed as $\mathrm{A} \rightarrow \mathrm{B} \rightarrow \mathrm{C} \rightarrow \mathrm{D} \rightarrow \mathrm{A}$ (Figure 11). Point $\mathrm{F}$ is the mounting 189 base point of cylinder 26, points G and G1 represent the positions of the rod tip of cylinder 26 at 190 the initial position and the position when end-effector reaches point C. $\theta$ is the angle of cylinder 19127 rotates about point $\mathrm{O}$ relative to its starting position during the trajectory from B to C. A five192 step implementation procedure was designed for this end-effector to capture and hold trellis 193 wires (Figure 12). The first step was the trellis wire being detected by bar 25 and switch S14 was 194 closed when the platform moved forward. For the following steps, which will be described in 195 detail correspondingly to the trajectory of $\mathrm{A} \rightarrow \mathrm{B} \rightarrow \mathrm{C} \rightarrow \mathrm{D} \rightarrow \mathrm{A}$. 


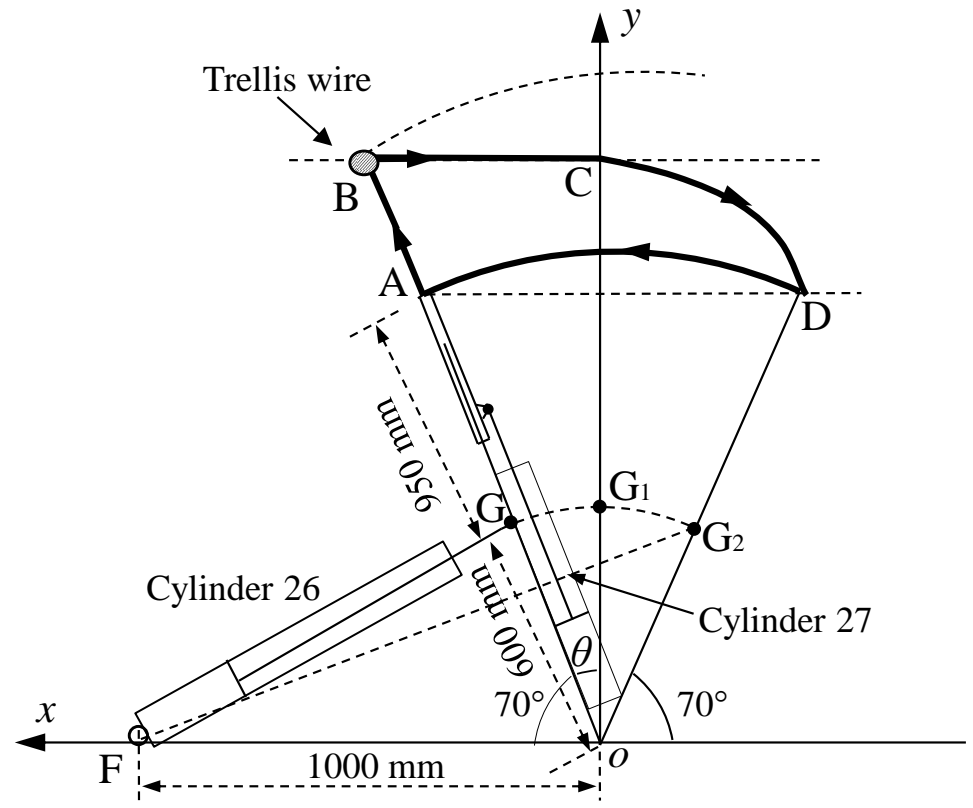

Figure 11. Illustration of the trajectory of trellis wire capturing operation

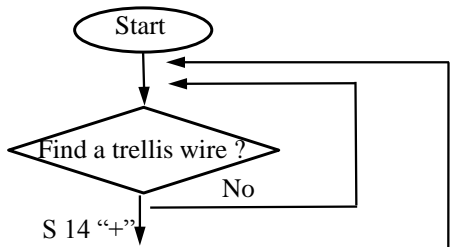

Go up to get the wire into desired position

Grasp and hold the wire

Delay $0.2 \mathrm{~s}$

Move the end-effector

horizontally backward

$\mathrm{S} 13$ "+"

Move the end-effector forward

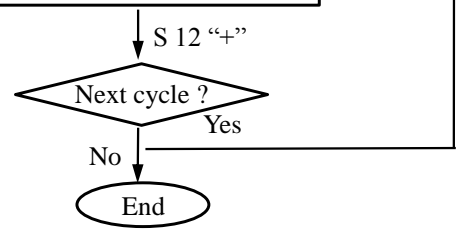

199 Figure 12. The control flowchart of trellis wire capturing end-effector. The symbol "+" indicates 
retracting speeds $v_{\mathrm{e} 2}$ and $v_{\mathrm{r} 2}$. The velocities of cylinders 26 and 27 are the average values of velocity in the corresponding actions.

In Segment $\mathbf{A} \rightarrow \mathbf{B}$ (step two) of the wire capturing trajectory, the end-effector was pushed upward as cylinder 27 being extended until the wire was captured and held firmly by the graspers 16 and 17 while the platform 28 was at a constant forward moving in the positive direction of $x$ axis. When the end-effector reached point B, the switch S15 was closed (S15 "+") by the trellis wire. It energized the solenoids to extend the cylinders 14 and 15 to hold the trellis wire firmly. The motions of cylinder 27 and the mobile platform 28 could be expressed using the following equations:

$$
\begin{aligned}
& v_{e 2}=l_{A B} / t_{1} \\
& l_{1}=v_{f} \cdot t_{1}
\end{aligned}
$$

where, $l_{\mathrm{AB}}$ is the extending distance of cylinder $27\left(l_{\mathrm{AB}}=250 \mathrm{~mm}\right.$ in this laboratory scale test $) ; t_{1}$ is the actuating duration for following this trajectory segment; and $l_{1}$ is the mobile platform forward moving distance from the time the trellis wire being detected to the time the trellis wire being firmly grasped. Due to the fixed location of the trellis wire, this distance $l_{1}$ should be equal to the horizontal distance from the touch point on detecting arm to the location of the trellis wire was grasped ( $l_{1}=200 \mathrm{~mm}$, based on the dimension of the robot). The velocity of the cylinder should be adjusted according to the speed of the mobile platform.

In Segment $\mathbf{B} \rightarrow \mathbf{C}$ (step three), $0.2 \mathrm{~s}$ delay was applied after S15 closing to ensure the trellis wire was hold firmly. Then, the end-effector was moving backward horizontally from point $\mathrm{B}$ to $\mathrm{C}$ by the combination actions of the cylinder 26 extending to compensate for the forwarding motion of the platform and the cylinder 27 retracting to compensate the height change during rotation of the end-effector relative to point $\mathrm{O}$. When the end-effector reached 
226 point $\mathrm{C}$, the graspers were ready to release the wire as the knot being completed. During the knot

227 tying process, the relative velocity between the end-effector and the trellis wire was zero

228 theoretically. The motion equation of this process could be expressed as follow:

229

$$
\left\{\begin{array}{c}
v_{f} \cdot t_{2}=l_{B C} \\
v_{r 2} \cdot t_{2}=l_{O B} \cdot \sin \left(70^{\circ}+\theta\right)-l_{O B} \cdot \sin 70^{\circ} \\
v_{e 1} \cdot t_{2}=l_{F G_{1}}-l_{F G}=\sqrt{l_{O F}^{2}+l_{O G_{1}}^{2}-2 l_{O F} l_{O G_{1}} \cos \left(70^{\circ}+\theta\right)}-l_{F G}
\end{array}\right.
$$

230 where, $t_{2}$ is actuating duration of moving the end-effector from $\mathrm{B}$ to $\mathrm{C}(\mathrm{s}) ; l_{\mathrm{FG}}, l_{\mathrm{OF}}$, and $l_{\mathrm{OG} 1}$ are

231 the dimensions of the end-effector (as denoted in Figure 13) (mm). In the designed end-effector, $232 \theta=20^{\circ}$.

233 In Segment $\mathbf{C} \rightarrow \mathbf{D}$ (step four), the end-effector is moving backward and downward 234 simultaneously with extending of the cylinder 26 and retracting of the cylinder 27. Cylinder 26 235 will stop until the switch 13 is on (S13 “+”), and the same to cylinder 27 when the switch 10 is 236 on (S10 “+").This segment of trajectory would be considered as finished when the switch 13 237 being turned on (S13 “+”). In this trajectory segment, the rotating angle of the end-effector 238 around point $\mathrm{O}$ was kept around $20^{\circ}$, the same as that in the segment $\mathrm{B}$ to $\mathrm{C}$. Therefore, the 239 duration of this segment $t_{3}$ was the same as the duration $t_{2}$ of the segment from $B \rightarrow C$.

$$
t_{3}=t_{2}
$$

241 where, $t_{3}$ is the duration for the trellis wire capturing and positioning end-effector trajectory from 242 C to D (s). In Segment $\mathbf{D} \rightarrow \mathbf{A}$ (step five) cylinder 26 was retracted to move end-effector forward 244 back to its initial position. The movement of this segment could be described using the follow 245 equation:

$$
v_{r 1} \cdot t_{4}=\sqrt{l_{o F}^{2}+l_{o G_{2}}^{2}-2 l_{o F} l_{o G_{2}} \cos 110^{\circ}}-l_{F G}
$$


247 where, $t_{4}$ is the duration for driving the end-effector from $\mathrm{D}$ to $\mathrm{A}(\mathrm{s})$; and $l_{\mathrm{OG} 2}$ is the dimension of 248 the end-effector (as denoted in Figure 13) (mm).

The forward distance of the mobile platform in the duration of one complete twining 250 cycle is expressed in equation (7). This distance will tell us whether the twining cycle could be 251 finished before the robot reaches the next trellis wire in the hop field.

$$
L=\left(t_{1}+t_{2}+t_{3}+t_{4}\right) \times v_{f}
$$

253 where, $L$ is the forward distance of the mobile platform during one complete twining cycle $(\mathrm{mm})$.

\subsubsection{Prototype Integration and Robot Control}

A laboratory scale research platform of integrated string twining robot was developed and

257 of the developed robot is about $2 \mathrm{~m}$ in length, $1.5 \mathrm{~m}$ in width, and $1.6 \mathrm{~m}$ in height. The overall height could reach up to $2 \mathrm{~m}$ when the cylinder 27 extends.

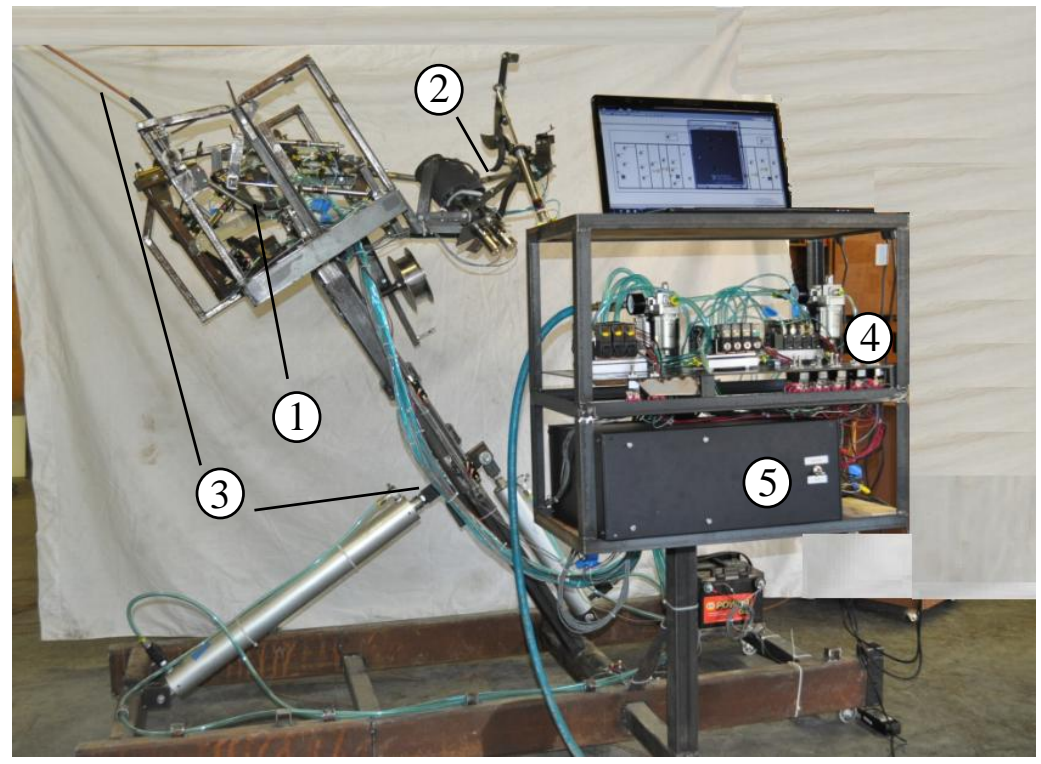

Figure 13. A fabricated mobile research platform of the twining robot. This robot was 

environment (version 2010, National Instruments, Austin, TX).

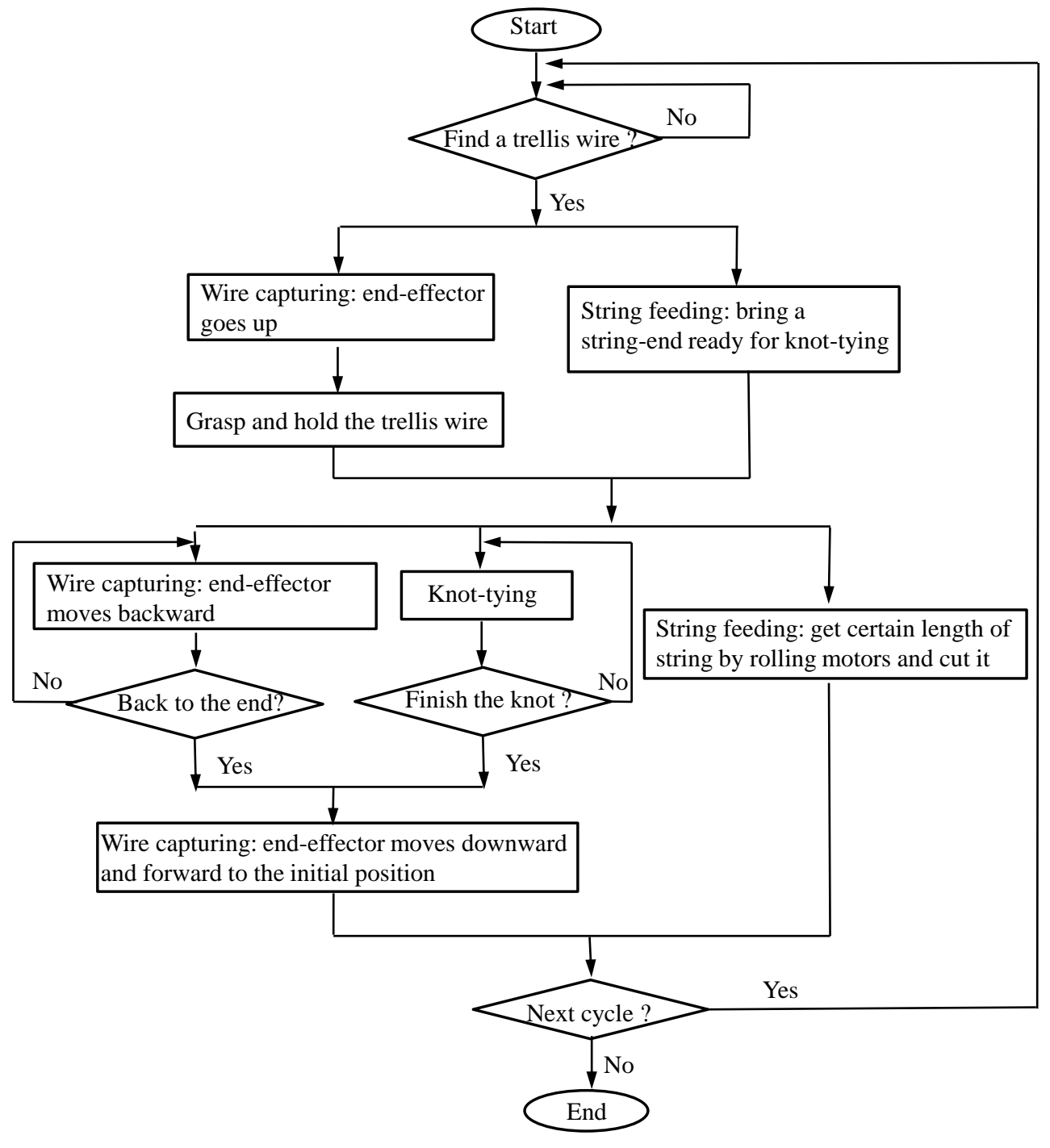

Figure 14. Control flowchart of the twining robot system 
As depicted in the control flowchart, a cycle of twining procedure starts as the detecting

272 bar senses the target trellis wire. Once found, the wire capturing end-effector moves up to

273 capture and hold the trellis wire. At the same time, the string feeding end-effector is also turned

274 on to deliver the string end to the start point of knot tying procedure. Once the trellis wire has

275 been grasped firmly, the knot tying end-effector starts the knotting and the trellis wire capturing

276 end-effector is operated as designed trajectories. Meanwhile, the string feeding end-effector

277 starts to rotate the roller pair to pull the required length of string and cut it. After a knot has been

278 tied, the wire capturing end-effector releases the wire and quickly reposition the end-effectors

279 back to their initial positions to make it ready for the next twining cycle.

\subsection{Experimental Validation}

To evaluate the performance of the concept-proof robotic mechanism and associated

282 control logic developed in this study, a series of functionality tests of all individual end-effectors

283 and the integrated system were conducted in a laboratory environment. Before the experiments,

284 the velocities of each cylinder were tuned manually according to the platform forward speed and

285 the procedure of twining process. To mimic the field working condition, two over-head trellis

286 wires were set up in the laboratory with $2.2 \mathrm{~m}$ apart and $2 \mathrm{~m}$ height. In future field test, the robot

287 will be mounted on a certain height frame to reach the required height of $5.5 \mathrm{~m}$.

288 Total 50 runs of string feeding tests were conducted to evaluate the string feeding end-

289 effector for the capability of producing $6 \mathrm{~m}$ of strings. The function test of wire capturing end-

290 effector was conducted to evaluate the performance of this end-effector in achieving the 291 designed procedure and trajectory. Two series of trellis wire capturing tests were conducted 292 under a platform forward speed of 0.19 and $0.25 \mathrm{~m} \cdot \mathrm{s}^{-1}$. The integrated robotic twining test was 293 conducted also in laboratory environment to evaluate the entire twining process by integrating all 
294 of three end-effectors as one system. All of end-effectors were controlled to work coordinately

295 following the control flowchart depicted in Figure 15. To verify the performance and reliability

296 of the twining robot, a total of 100 twining cycles were conducted in the laboratory setup with

297 forward speed of $0.19 \mathrm{~m} \cdot \mathrm{s}^{-1}$. For each twining, the cycle time started when the robot detected the

298 trellis wire and ended when the robotic device released the captured trellis wire after completing

299 the knot tying. A high speed camera (EX-FH25, CASIO Computer, Tokyo, Japan) was used to

300 record the twining process with a record speed of 120 frames per second. The duration of each

301 action in this twining process was determined from the number of frames recorded in the video.

302 The drive distance of the platform was obtained by the real cycle time multiplying the forward

303 speed of the platform.

$304 \quad 3$ Results and Analysis

305 3.1 String Feeding Test

306 Over a total of 50 testing runs, the length of the cut strings was $6.04 \pm 0.15 \mathrm{~m}$, with the 307 maximum length of $6.20 \mathrm{~m}$ and minimum length of $5.93 \mathrm{~m}$. As the target length was $6.0 \mathrm{~m}$, these 308 results were considered acceptable. A modified control method based on the counting of roller 309 revolutions instead of running duration of the motors might improve the precision in string 310 length control.

3113.2 Trellis Wire Capturing Functionality Test

312 For trellis wire capturing functionality test, there was no data to show the positions of the 313 end-effector in operation, however, based on the observation, the developed end-effector 314 functionally finished the required work. The measured total time and moving distance for one 315 twining cycle are listed in Table 1 . Table 1 also provides the calculated average extending and 316 retraction velocities of cylinders 26 and 27 and time for required for each segment of the trellis 
317 wire capturing end-effector motion $\mathrm{A} \rightarrow \mathrm{B} \rightarrow \mathrm{C} \rightarrow \mathrm{D} \rightarrow \mathrm{A}$. Based on this theoretical analysis, it 318 would take 10.2 or $8.2 \mathrm{~s}$ to complete a cycle of trajectory when the platform moved forward at 3190.19 or $0.25 \mathrm{~m} \cdot \mathrm{s}^{-1}$. The corresponding moving distance was 1.9 or $2.1 \mathrm{~m}$, respectively, for those 320 conditions.

321 Table 1. Calculated cylinder velocities in trellis wire capturing end-effector and segment time 322 under different platform forward moving speeds

\begin{tabular}{|c|c|c|c|c|c|c|c|c|c|c|c|}
\hline \multirow{2}{*}{$\begin{array}{l}\text { Platform } \\
\text { speed } \\
\left(\mathrm{m} \cdot \mathrm{s}^{-1}\right)\end{array}$} & & \multicolumn{4}{|c|}{ Cylinder speed $\left(\mathrm{m} \cdot \mathrm{s}^{-1}\right)$} & \multicolumn{4}{|c|}{ Segment time (s) } & \multirow{2}{*}{$\begin{array}{l}\text { Total } \\
\text { time } \\
(\mathrm{s})\end{array}$} & \multirow{2}{*}{$\begin{array}{l}\text { Drive } \\
\text { distance } \\
(\mathrm{m})\end{array}$} \\
\hline & & $v_{\mathrm{e} 1}$ & $v_{\mathrm{r} 1}$ & $v_{\mathrm{e} 2}$ & $v_{\mathrm{r} 2}$ & $\underset{t_{1}}{\mathrm{~A} \rightarrow \mathrm{B}}$ & $\underset{t_{2}}{\mathrm{~B}} \rightarrow \mathrm{C}$ & $\underset{t_{3}}{\mathrm{C} \rightarrow \mathrm{D}}$ & $\underset{t_{4}}{\mathrm{D}} \rightarrow \mathrm{A}$ & & \\
\hline \multirow[t]{2}{*}{0.19} & Calculated & 0.06 & 0.12 & 0.30 & 0.03 & 1.0 & 3.6 & 3.6 & 2.0 & 10.2 & 1.9 \\
\hline & Measured & - & - & - & - & 1.2 & 3.9 & 3.8 & 2.3 & 11.2 & 2.1 \\
\hline \multirow[t]{2}{*}{0.25} & Calculated & 0.08 & 0.12 & 0.37 & 0.04 & 0.8 & 2.7 & 2.7 & 2.0 & 8.2 & 2.1 \\
\hline & Measured & - & - & - & - & 1.1 & 3.1 & 2.9 & 2.2 & 9.3 & 2.3 \\
\hline
\end{tabular}

The laboratory tests showed that it took 11.2 or $9.3 \mathrm{~s}$ in average for the testing robotic wire capturing end-effector to complete one cycle when the platform was moving forward at a speed of 0.19 and $0.25 \mathrm{~m} \cdot \mathrm{s}^{-1}$ respectively, roughly $10 \%$ slower than the theoretical cycle time.

326 Such additional time required to compete the cycle in actual tests was mainly attributed to the 327 delay of the pneumatic cylinders starting to response due to the small size the air pump, 328 especially for cylinders 26 and 27 due to their large sizes. Under such additional time required to 329 compete one twining cycle, the robot platform drove forward 2.1 and $2.3 \mathrm{~m}$ respectively, about $0.2 \mathrm{~m}$ more in average than theoretically calculated distance. In typical commercial hop fields in 331 Washington, the trellis spacing is approximately $2.2 \mathrm{~m}$. The laboratory validation test revealed 332 that the developed research platform with current configuration could continuously complete an 333 entire twining cycle as the platform moved from one trellis to the next under the speed of 0.19 $334 \mathrm{~m} \cdot \mathrm{s}^{-1}$. However, when the speed was $0.25 \mathrm{~m} \cdot \mathrm{s}^{-1}$, the drive distance of the platform was 2.3 meter 335 which is over the $2.2 \mathrm{~m}$ trellis wires space in the field. Therefore, in the integrated robotic 336 twining test, we only conducted the test at the speed of $0.19 \mathrm{~m} \cdot \mathrm{s}^{-1}$. 


\subsection{Integrated Robotic Twining Test}

Three out of 100 integrated robotic twining tests were not completed during the entire twining cycle, and resulted in a successful rate of 97\%. Among those unsuccessful trials, one was caused by the trellis wire capturing end-effector missed the targeted trellis. The other two failures were caused by the knot tying end-effector failing to finish the knot. The most likely cause to both failures was the excessive vibration during cylinders acceleration and deceleration, resulting the components veering off the planned path during movement. Low manufacturing precision could be a reason for this vibration as almost all the non-standard components were manually made just for the purpose of proving the concept. A high precision in components fabrication might reduce the unexpected high vibration level and improve the success rate of the robotic twining operation.

\section{Conclusions}

A proof of concept string twining robot suitable for Washington high-trellis hop production has been designed, fabricated and tested in this study. The following major conclusions were obtained from those tests.

1) The conceptual string feeding end-effector reliably fed the strings continuously with the required length.

2) It was visually observed that the conceptual trellis wire capturing end-effector functionally achieved the designed function by accomplishing the designed trajectories.

3) The integrated robot system synchronized all end-effectors to finish the entire process, resulted in a successful rate of $97 \%$ when the platform forward speed was set at $0.19 \mathrm{~m} \cdot \mathrm{s}^{-1}$.

Based on observation of manual twining operations performed by professional hop field workers in the twining seasons of 2011 and 2012, a skilled worker could tie one knot in 7 s, 
360 which was about $150 \%$ faster than the developed prototype. Based on the results obtained from

361 this laboratory proof of concept tests, there is potential for increasing the speed of actuating

362 cylinders to reduce the time for finishing a cycle. Additionally, up to four knot tying end-

363 effectors can share one mobile platform and work synchronously to tie multiply knots at one run,

364 which would improve productivity. In our future work, the developed twining robot will be

365 modified with a higher fabrication precision, and a field test will be conducted on this developed

366 twining robot.

367 Acknowledgments

368 The research presented in this paper was partially supported by S.S. Steiner, Inc., the 369 United States Department of Agriculture (USDA)'s Hatch Funds (WNP0745 and WPN00728), 370 Washington State University Agricultural Research Center (WSU-ARC) and WSU Center for 371 Precision and Automated Agricultural Systems (CPAAS). Any opinions, findings, and 372 conclusions expressed in this paper are those of the authors, and do not necessarily reflect the 373 views of S.S. Steiner, Inc., USDA or WSU.

\section{References}

375 Gobor, Z., and Fröhlich, G. (2010). Automated fastening of the support wire in high trellis of hop. $376 \quad$ Landtechnik, 65(4), 283-285.

377 Gobor, Z., Fröhlich, G., Soller, H., and Portner, J. (2012). First operating experiences with a 378 prototype for automated attaching of the supporting strings that the hop vines grow on in high-trellis hop gardens. International Conference of Agricultural Engineering, CIGRAgeng2012, July 8-12, 2012. Valencia, Spain. tying in hops production. Transactions of the ASABE, 55(5): 1667-1673. 
383 He, L., Zhang, Q., and Charvet, H. (2013). A knot-tying end-effector for robotic hop twining. Biosystems Engineering, 114(3): 344-350.

385 Keller Swartwood Engineering, Inc. (2003). The design manual for hop twining machine. 386 Unpublished equipment design manual, Aurora, OR.

387 Schoonheere, M. J., K. Naeyaert, and J. A. E. Vande Ryse. (2007). Double knotting system for 388 an agricultural baler. U.S. Patent No. 7,296,828 B2.

389 Singhatat, W., and Corporation, L. (2004). Intracorporeal Knot Tier. U.S. Patent No. 6,716,224 B2.

391 Thomas, D., and Jenkins, S. (2003). Automatic knot-tying Machine. U.S. Patent No. 6,641,181 B2.

393 Torres, M., and Riehm, G. (2003). Automatic knot-tying device. U.S. Patent No. 6,648,378 B1.

394 USDA-NASS. (2011). National Hop Report. Washington, D.C.: USDA National Agricultural 395 Statistics Service (NASS). Available at: www.nass.usda.gov. Accessed 12 December 396 2012.

397 WSDLI. (2002). Hop growing, harvesting and processing (Update). Olympia, WA.: Washington 398 State Department of Labor \& Industries. Available at: www.lni.wa.gov. Access on 25 December 2012. 